\title{
The dialectics of infinitism and coherentism: Inferential justification vs. holism and coherence
}

\author{
Frederik Herzberg \\ Copyright notice: The final publication, in Synthese, 191 (2014), no. 4, pp. \\ 701-723, is available at link.springer.com, doi:10.1007/s11229-013-0273-5
}

\begin{abstract}
This paper formally explores the common ground between mild versions of epistemological coherentism and infinitism; it proposes - and argues for - a hybrid, coherentistinfinitist account of epistemic justification.

First, the epistemological regress argument and its relation to the classical taxonomy regarding epistemic justification - of foundationalism, infinitism and coherentism - is reviewed. We then recall recent results proving that an influential argument against infinite regresses of justification, which alleges their incoherence on account of probabilistic inconsistency, cannot be maintained. Furthermore, we prove that the Principle of Inferential Justification has rather unwelcome consequences formally resembling the Sorites paradox - as soon as it is iterated and combined with a natural Bayesian perspective on probabilistic inferences. We conclude that strong versions of foundationalism and infinitism should be abandoned. Positively, we provide a rough sketch for a graded formal coherence notion, according to which infinite regresses of epistemic justification will often have more than a minimal degree of coherence.

Key words: epistemic justification; coherentism (epistemology); infinitism (epistemology); foundationalism (epistemology); regress; Principle of Inferential Justification; probabilism; Bayesianism; coherence
\end{abstract}

This work was financially supported by the Alexander von Humboldt Foundation through a Visiting Fellowship of the Munich Center for Mathematical Philosophy at Ludwig Maximilian University of Munich. I am deeply grateful to Professor Hannes Leitgeb for very helpful discussions, as well as to Professor Jeanne Peijnenburg and an anonymous referee for many insightful comments that helped to improve the paper substantially. 


\section{Contents}

1. Introduction 3

2. Foundationalism, infinitism and coherentism as responses to the regress problem

3. Structural origins, weakenings and mutual compatibility of foundationalism, infinitism and coherentism

4. A formal probabilistic framework for analysing belief systems and their epistemic justification

5. In defense of (weak) infinitism: The consistency of infinite probabilistic regresses

6. Common ground between strong foundationalism and strong infinitism: The Principle of Inferential Justification 14

7. Problematic consequences of Bayesian variants of the Principle of Inferential Justification

8. A dilemma for Bayesian foundationalists and infinitists 20

9. Toward a solution: Reviving coherentism 21

10. Conclusion

References 


\section{Introduction}

Philosophical accounts of the structure of reasons and thus of epistemic justification are typically said to fall into one of three categories — viz. foundationalism, infinitism and coherentism. This taxonomy of non-skeptical positions regarding epistemic justification has been around for more than two millennia; it is closely related to the notion of a regress of reasons and exhibits - at least historically - a certain bias in favour of foundationalism. For, it was arguably! - in defense of foundationalism that Aristotle gave, in his Posterior Analytics (Book I, Part 3) in the middle of the fourth century BC, an early version of the regress argument: "since the regress must end in immediate (" $\alpha \mu \varepsilon \sigma \alpha)$ truths, those truths must be indemonstrable ( $\alpha \nu \alpha \tau o ́ \delta \varepsilon เ \chi \tau \alpha)$ )" (in the translation by G.R.G. Mure [2]; original Greek terms inserted by this author). In response to the popular foundationalist epistemological reading of the Aristotelian argument, infinitists would deny that there "must [be an] end" to the regress at all, and coherentists would remark that epistemic justification should not be conceived of as a propositional or doxastic regress - in the sense of a linearly ordered structure of reasons - in the first place.

Even to this very day, the threefold classification of non-skeptical accounts of epistemic justification as foundationalist, infinitist or coherentist enjoys widespread acceptance - so much that coherentism is often defined, through the regress argument, simply as the third option, as it were, of epistemic justification (cf. e.g. Spohn [61], Bergmann [5] or the encyclopedia article by Kvanvig [40]). Within such a presentation, coherentism is basically presented as the only viable non-skeptical alternative to foundationalism and infinitism and by definition opposed to the latter two positions. As almost everywhere in philosophy, there are also alternative views. Richard Swinburne, for example, opines that "there is no good reason to suppose that the various [...] accounts [of epistemic justification] are in general rival accounts, one at most of which is the true account, of a univocal concept". Also, Audi's "moderate foundationalism" [3. p. 233] does not insist on a strictly linear structure of reasons and thereby leaves room for coherence considerations and thus for a middle ground between coherentism and foundationalism.

In this article, we attempt to present a formal defence of the mutual compatibility of mild versions of coherentism and infinitism, while arguing that strong versions of foundationalism and infinitism are faced with important difficulties. First, we shall distinguish and define different versions of foundationalism, infinitism and coherentism - and study their relation to the epistemological regress argument. Then, we shall recall that there is a sense in which probabilistic infinite 
regresses of epistemic justification satisfy a minimal requirement of coherence, viz. probabilistic consistency, so that an attempt at blending infinitism and coherentism in a probabilistic setting is not automatically doomed to failure. We will also see, however, that a certain common characteristic of pronounced infinitist and foundationalist positions, viz. the Principle of Inferential Justification, is actually untenable in a probabilistic setting. We conclude that those versions of infinitism and foundationalism which are built on the Principle Inferential Justification (for short: strong infinitism and strong foundationalism) should be rejected. Positively, we describe the outlines of a formal graded coherence notion for conditional degrees of beliefs (understood as conditional probability assignments, inducing sets of probability measures) and show that certain infinite regresses of epistemic justification do possess a reasonable degree of coherence.

In brief, this article will furnish a formal argument that there is (i) a positive common ground between coherentism and mild versions of infinitism - beyond the mere opposition to foundationalism — and (ii) good reasons to set one's epistemological stakes there. This may come as a surprise, since Peter Klein co-authored formal papers attacking coherentism (with Warfield [38, 39]) not long before setting out to publish his well-known papers reviving infinitism (e.g. [33, 34, 35]). But one may read Klein and Warfield [38, 39] as merely criticising a certain non-essential way of formalising coherentism, not the positive core tenets of coherentism itself.

The paper is structured as follows. In Sections 2 and 3 , we review the classical threefold classification of accounts of epistemic justification - foundationalism, infinitism and coherentism - and explore possibilities of reconciliation. In Section 4, we set up a formal framework in which we can analyse the probabilistic consistency of belief systems in general and of infinite regresses of probabilistic justification (for short: probabilistic infinite regresses) in particular. In Section 5, we shall review recent findings about the probabilistic consistency of infinite regresses. In Sections 6 and 7, we shall argue that a consistent probabilistic regress of reasons, be it finite or infinite, may not in itself provide epistemic justification; the Principle of Inferential Justification has bizarre consequences, formally resembling the Sorites paradox, in a probabilistic framework. We conclude (in Section 8) that the Principle of Inferential Justification should be abandoned. Finally, we propose to return to the search for a coherence theory of epistemic justification - one in which some aspects of infinitism can be retained. In Section 9, we sketch the basics of a graded coherence notion that formalises BonJour's [6] account of coherence. Section 10 concludes.

In the choice of our formal framework, we follow many contemporary epistemologists (including infinitists such as Peijnenburg and Atkinson [51]) in adopting probabilism ('Bayesianism'), i.e. the 
view that doxastic states are graded and have the formal properties of probability assignments.

\section{Foundationalism, infinitism and coherentism as responses to the regress problem}

A classical argument for foundationalism based on the epistemological regress problem is commonly attributed to Aristotle's Posterior Analytics (Book I, Part 3). Whether Aristotle actually intended his reasoning to be understood as a defence of epistemological foundationalism (against Plato) or rather of a certain method of instruction is a historical, exegetical question - which depends on the interpretation of the term "demonstration" ( $\dot{\alpha} \pi o ́ \delta \varepsilon เ \xi ı \zeta)$ in Aristotle's Organon - to which variant responses have been offered (cf. Irwin [30, p. 139] or Rescher [57, p. 702] for the former view and Barnes [4. p. 138] for the latter; Stump [62] offers a more detailed account of the rise of foundationalism through a certain reading of Aquinas and Aristotle). The present paper, however, has a 'systematic' rather than 'historical' concern. We shall therefore henceforth ignore the historical question of Aristotle exegesis and confine ourselves to a discussion of some aspects of the argument for foundationalism that one obtains when following the epistemological interpretation of Aristotle's Posterior Analytics.

The epistemological regress argument can be formally reconstructed as follows (with propositional justification replaced by doxastic justification). The first and pivotal step is to note that the following set of premises is inconsistent: [ $^{1}$

(1) ['All justified beliefs are based on a non-repeating chain of reasons (of length zero in the case of immediate justification).'] If a subject $\left.\right|^{2} \mathcal{J}$ is justified in believing a proposition $S_{0}$, then there exists a sequence $\left\langle S_{1}, S_{2}, \ldots\right\rangle$ of other propositions (of zero, finite non-zero or infinite length) such that

(a) for all $k>0, \mathcal{J}$ is justified in believing $S_{k}$, and $S_{k} \neq S_{0} !^{3}$

(b) for all $k, S_{k+1}$ is a reason for $S_{k}$, and

(c) if $\left\langle S_{1}, S_{2}, \ldots\right\rangle$ is of finite length (say $n$ ), then it is maximal in the sense that there is no proposition $S^{\prime}$ such that J justifiedly believes $S^{\prime}$ and $S^{\prime}$ is a reason for $S_{n}$.

\footnotetext{
${ }^{1}$ I am very grateful to an anonymous referee for pointing out several weaknesses in an earlier version of this section and the following one; any remaining errors are mine.

${ }^{2}$ Mnemonics: The letter J abbreviates 'individual'; $S$ abbreviates 'sentence'.

3 The second half of this requirement is, strictly speaking, redundant — because we have already demanded $S_{1}, S_{2}, \ldots$ to be propositions other than $S_{0}$ - and has only been inserted for clarification.
} 
Such a sequence $\left\langle S_{1}, S_{2}, \ldots\right\rangle$ is called a chain of reasons for $S_{0}$, and J is said to be justified in believing $S_{0}$ on the basis of the chain of reasons $\left\langle S_{1}, S_{2}, \ldots\right\rangle$.

(2) I'If there are justified beliefs, then at least some of them are based on finitely many reasons.'] If there are a subject I' and a proposition $S_{0}^{\prime}$ such that $\mathcal{J}^{\prime}$ is justified in believing $S_{0}^{\prime}$, then there also is a subject $\mathcal{J}$ and a proposition $S_{0}$ such that $\mathcal{J}$ is justified in believing $S_{0}$ on the basis of a finite chain of reasons.

(3) ['There are justified beliefs.'] There exists a subject $\mathcal{J}$ and a proposition $S_{0}$ such that $\mathcal{J}$ is justified in believing $S_{0}$.

(4) ['There are no immediately justified beliefs.'] There is no subject J and no proposition $S_{0}$ such that J would be justified in believing $S_{0}$ on the basis of a chain of reasons of length zero.

The circumscription of Premise 1 is correct, because even though the Premise prima facie only requires $S_{1}, S_{2}, \ldots$ to be distinct from $S_{0}$ (second half of part 1a), its iteration implies that $S_{0}, S_{1}, \ldots$ are all (pairwise) distinct.

The inconsistency proof for these premises is straightforward:

Proof of the inconsistency of Premises 1, 2, 3, 4. Premise 3 and Premise 1 imply that there is some subject $\mathcal{J}$ and a proposition $S_{0}$ such that $\mathcal{J}$ is justified in believing $S_{0}$ on the basis of some chain of reasons $\left\langle S_{1}, S_{2}, \ldots\right\rangle$. By Premise 2, J and $S_{0}$ may be chosen in such a way that $\left\langle S_{1}, S_{2}, \ldots\right\rangle$ is finite, say of length $n$.

Then, on the one hand, J is justified in believing $S_{n}$. Hence there must be, by Premise 1, a chain of reasons for $S_{n}$. Now, suppose that this chain of reasons were not of length zero. Then, concatenating $\left\langle S_{1}, S_{2}, \ldots\right\rangle$ with this new chain of reasons would yield a new, longer chain of reasons for $S_{0}$ — which would contradict the maximality requirement (part 1c) of Premise 1. Thus, we have found an individual $\mathcal{J}$ and a proposition $S_{n}$ such that $\mathcal{J}$ believes $S_{n}$ on the basis of a chain of reasons of length zero. This, however, directly contradicts Premise 4

For illustration, first consider a simple example of circular reasoning. Suppose a subject $\mathcal{J}$ has just two beliefs, $S_{0}$ and $S_{1}$, and she reports that $S_{1}$ is her reason for belief in $S_{0}$, and $S_{0}$ is her reason for belief in $S_{1}$. Now, on the one hand, the one-entry sequence $\left\langle S_{1}\right\rangle$ is clearly not a chain of reasons (because $\mathcal{J}$ has a reason for believing $S_{1}$, viz. $S_{0}$, whence the maximality clause $1 \mathrm{c}$ of Premise 1 denies $\left\langle S_{1}\right\rangle$ the status of a chain of reasons). On the other hand, no sequence in which $S_{0}$ occurs can be a chain of reasons for $S_{0}$ because this would violate the requirement that the proposition to be justified may not be adduced as a reason for itself (second half of clause $1 \mathrm{a}$ of Premise 1 . ruling out petitio principii). By the same argument, $S_{1}$ may not be a reason for itself, so that the demand of part $1 \mathrm{~b}$ of Premise 1 cannot be 
met by any sequence that consists of repeated entries of $S_{1}$ only and has length greater than one. Hence, Premise 1 actually denies that the subject in question has any justified beliefs at all.

Next, consider an example of infinitist justification. Suppose that a subject $\mathrm{J}^{\prime}$ believes proposition $S_{0}$ and reports another proposition, $S_{1}$, as a reason for $S_{0}$, yet another reason, $S_{2}$, for $S_{1}$ etc. ad infinitum. Then Premise 1 is clearly satisfied, and there is no contradiction to the other Premises, even though Premise 2 requires that not all cases of justified belief have this form (hence the inconsistency of the Premises).

Finally, let us look at an example of foundationalist justification. Reconsider subject $\mathcal{J}$ from above with her two beliefs, $S_{0}$ and $S_{1}$, and suppose now that she reports that $S_{1}$ is her reason for belief in $S_{0}$, and that her chain of reasons for $S_{1}$ is the sequence of length zero. This scenario satisfies Premise 1 but directly contradicts Premise 4.

Let us call the problem posed by the inconsistency of those prima facie not entirely implausible premises the regress problem.

The foundationalist claims (with Aristotle) that all premises except Premise 4 are self-evident. The conclusion is that Premise 4 is actually false. Therefore, immediately justified (foundational) beliefs do exist.

This reasoning can, however, be turned into an argument for skepticism by claiming that it is in fact not Premise 4, but Premise 3 . which is dubious and therefore should be jettisoned. Equally, if one can make the case that the most questionable assumption is Premise 2, one obtains an argument for infinitism. Finally, one can attack Premise 1 as the most problematic; this yields an - even though rather artificial - argument for coherentism.

In this vein, the three classical non-skeptical epistemological positions - foundationalism, infinitism, and coherentism - can be introduced as rival responses to the problem of regresses of epistemic justification (as, for instance, in Klein [37] or Kvanvig [40]). There can thus be no question of reconciling any two of those positions.

However, especially in light of the somewhat artificial coherentist response to the regress problem, it is perhaps more plausible to say that these three positions come from fundamentally different intuitions about the very structure of epistemic justification - whether justification is a property of beliefs or belief systems and, if the former, whether there are foundational beliefs (that is, beliefs which are noninferentially or immediately justified).

\section{Structural origins, weakenings and mutual compatibility of foundationalism, infinitism and coherentism}

In this section, we (i) show how the germs of foundationalism, infinitism and coherentism can already be found in certain fundamental views about the structure of epistemic justification, (ii) distinguish weaker and stronger versions of foundationalism, infinitism and 
coherentism, and explore the compatibility of weaker positions of foundationalism, infinitism and coherentism, (iii) formulate weak versions of foundationalism, infinitism and coherentism that treat epistemic justification as a matter of degree.

\subsection{Germs of foundationalism, infinitism and coherentism.}

We can distinguish two basic and common intuitions about epistemic justification (without claiming to be exhaustive of all possibilities): On the one hand, one can view epistemic justification as a purely "local" relation, as it were, so that a single proposition is either immediately justified by recourse to another single proposition. A variant of this position is to assent to a "local" character of epistemic justification in this sense, but to deny the possibility of immediate epistemic justification. On the other hand, there is some prima facie plausibility in viewing epistemic justification holistically, i.e. as a "global" property - a property which pertains to whole sets of propositions.

One can summarise these basic intuitions in the following theses:

(Strong pre-Foundationalism) A subject $\mathcal{J}$ is justified in believing a proposition $S_{0}$ if and only if $S_{0}$ is either foundational or $\mathcal{J}$ believes another proposition $S_{1}$ such that $S_{1}$ is a reason for $S_{0}$ and $\mathcal{J}$ is justified in believing $S_{1}$.

(Strong pre-Infinitism) A subject $\mathcal{J}$ is justified in believing a proposition $S_{0}$ if and only if $\mathcal{J}$ believes another proposition $S_{1}$ such that $S_{1}$ is a reason for $S_{0}$ and $\mathcal{J}$ is justified in believing $S_{1}$.

(Strong pre-Coherentism) A subject $\mathcal{J}$ is justified in believing a proposition if and only if it belongs to a set $\mathcal{S}$ of propositions such that $\mathcal{J}$ believes all propositions in $\mathcal{S}$ and $\mathcal{J}$ is justified in believing $\mathcal{S}$ as a whole.

Several observations are in order. First, it is common to view " $S_{1}$ is a reason for $S_{0}$ " as synonymous with " $S_{0}$ can be inferred from $S_{0}$ ", which allows for a re-formulation of the positions of Strong preFoundationalism and Strong pre-Infinitism. It then becomes clear that Strong pre-Infinitism and Strong pre-Foundationalism share an important common feature in assigning a pivotal rôle to inferential justification, but differ as to whether all justification is inferential. An alternative which downplays the rôle of inferential justification would be to replace " $S_{1}$ is a reason for $S_{0}$ " by " $S_{1}$ is held as a belief in virtue of $S_{0}$ "; this, however, comes at the cost of having to explicate what it means to assert to one proposition in virtue of another.

Second, the above theses of Strong pre-Infinitism and Strong pre-Foundationalism, even when thus reformulated, cannot be used immediately as definitions of what it means for a subject to be justified 
in believing a proposition, because the predicate 'justified' occurs both in the definiens and the definiendum. Third, Strong preFoundationalism reduces to Strong pre-Infinitism if one denies that there are any foundational propositions at all.

Strong pre-Infinitism, when reformulated as indicated above (replacing " $S_{1}$ is a reason for $S_{0}$ " by " $S_{0}$ can be inferred from $S_{0}$ "), naturally — by iteration — gives rise to a strong version of infinitism:

(Strong Infinitism) A subject $\mathcal{J}$ is justified in believing a proposition $S_{0}$ if and only if $\mathcal{J}$ believes other propositions $S_{0}, S_{1}, S_{2}, \ldots$ (ad infinitum), is justified in believing each of $S_{1}, S_{2}, S_{3}, \ldots$ and for all $k, S_{k}$ can be inferred from $S_{k+1}$.

In fact, Strong Infinitism is logically equivalent to Strong preInfinitism: Strong Infinitism obviously implies Strong pre-Infinitism, and the latter implies the former by a straightforward iteration.

Foundationalists would typically not merely assert to Strong preFoundationalism, but would also want to assert that the structure of reasons is a well-ordering, so that any set that is ordered by the relation "is a reason for" has a minimal and thus foundational element.

(Strong Foundationalism) A subject $\mathcal{J}$ is justified in believing a proposition $S_{0}$ if and only if $S_{0}$ is either foundational or $\mathcal{J}$ believes some other propositions $S_{1}, \ldots, S_{n}$ ( $n$ being a non-negative - and, to be sure, finite - integer), is justified in believing each of them and for all $k<n, S_{k}$ can be inferred from $S_{k+1}$ while $S_{n}$ is foundational.

The additional requirement - the demand that the reasons be well-ordered - is indispensable for deriving Strong Foundationalism from Strong pre-Foundationalism. The reason is that Strong Foundationalism and Strong pre-Foundationalism are not equivalent, although clearly the former implies the latter. In order to see that Strong Foundationalism cannot follow logically (without further hypotheses) from Strong pre-Foundationalism, note that Strong preFoundationalism does not demand that the set of foundational propositions be non-empty; Strong Foundationalism, however, asserts the existence of foundational propositions - and thereby contradicts Strong Infinitism — whenever there are any justified propositions. (In fact, if there are no foundational propositions, then Strong preFoundationalism is equivalent to Strong pre-Infinitism and thus to Strong Infinitism.)

But even Strong Foundationalism and Strong Infinitism cannot be used to define epistemic justification because the predicate 'justified' appears on both sides of the equivalence statements constituting those theses. For example, the position that a subject is epistemically 
justified in believing all propositions is consistent with Strong preFoundationalism, Strong pre-Infinitism and Strong Infinitism; if the subject in question believes all propositions including contradictions she may always point to any contradiction as a - possibly even foundational - "reason" due to the ex falso quodlibet. Also, the position that a subject is only epistemically justified in believing tautologies is consistent with Strong (pre-) Infinitism as well as with Strong Foundationalism, as long as only tautologies are deemed foundational. (For the compatibility with Strong pre-Infinitism and Strong Infinitism, note that there are infinitely many tautologies.)

3.2. Weaker versions of foundationalism, infinitism and coherentism. Their mutual compatibility. A version of infinitism that is slightly weaker than those presented so far can be obtained by dropping one half of the equivalence statement in the thesis of Strong Infinitism. A particularly cautious treatment of epistemic justification (which is true to Peter Klein's anti-dogmatist intentions) will retain the "only if" part:

(Weak Infinitism) A subject $\mathcal{J}$ is justified in believing a proposition $S_{0}$ only if $\mathcal{J}$ believes other propositions $S_{0}, S_{1}, S_{2}, \ldots$ (ad infinitum), is justified in believing each of $S_{1}, S_{2}, S_{3}, \ldots$ and for all $k, S_{k}$ can be inferred from $S_{k+1}$.

The definition of Strong pre-Coherentism can be used as a definition of justification of individual beliefs, at the cost of having to specify what justification of belief systems amounts to. As the name suggests, Coherentism understands this chiefly as a matter of coherence. In order to avoid having to call mere internal fiction epistemically justified, strong coherentists need to modify their position by requiring of epistemically justified belief systems that they be non-isolated, i.e. that they accommodate at least some external input. (Cf. e.g. BonJour [6] or the irenic, "ecumenical" treatment in Lehrer [41]). For a weaker version of coherentism - one which drops the "if" part in the thesis of Strong Coherentism ("necessity coherentism", cf. e.g. Murphy [47]) this modification is not needed:

(Strong Coherentism) A subject $\mathcal{J}$ is justified in believing a proposition if and only if it belongs to a set $\mathcal{S}$ of propositions such that $\mathcal{J}$ believes all propositions in $\mathcal{S}$ and $\mathcal{S}$ is coherent and non-isolated.

(Weak Coherentism) A subject $\mathcal{J}$ is justified in believing a proposition only if it belongs to a set $\mathcal{S}$ of propositions such that $\mathcal{J}$ believes all propositions in $\mathcal{S}$ and $\mathcal{S}$ is coherent.

On such an account, Weak Infinitism and Weak Coherentism are no longer mutually exclusive. This should not come as too much of a 
surprise because infinitism and coherentism share a common intuition — in addition to their opposition to foundationalism: Both positions tend to make the epistemic justification of individual propositions dependent on the epistemic justification of very large sets of other propositions. In infinitism, this is an obvious consequence of the infinite regress-like structure of epistemic justification assumed therein. But coherentism, too, is arguably strongest when used to describe the epistemic justification of very large belief systems (sometimes called "holistic varieties" of coherentism, e.g. in Kvanvig [40]).

3.3. Foundationalism, infinitism and coherentism for graded epistemic justification. If epistemic justification is a matter of degree, as many epistemologists think, cf. e.g. Roderick M. Chisholm [12] or Peter Klein [35, p. 138], a further modification is necessary. One then arrives at the following positions:

(Weak Graded Foundationalism) The degree to which a subject $\mathcal{J}$ is justified in believing a proposition $S_{0}$ is, ceteris paribus, increased by the extent to which $\mathcal{J}$ believes and is justified in believing some other propositions $S_{1}, \ldots, S_{n}$ ( $n$ being a non-negative and, to be sure, finite - integer) such that for all $k<n, S_{k}$ can be inferred from $S_{k+1}$ while $S_{n}$ is foundational; it is also increased by the strength of those inferences.

(Weak Graded Infinitism) The degree to which a subject $\mathcal{J}$ is justified in believing a proposition $S_{0}$ is, ceteris paribus, increased by the extent to which $\mathcal{J}$ believes and is justified in believing other propositions $S_{0}, S_{1}, S_{2}, \ldots$ (ad infinitum) such that for all $k, S_{k}$ can be inferred from $S_{k+1}$; it is also increased by the strength of those inferences.

(Weak Graded Coherentism) The degree to which a subject $\mathcal{J}$ is justified in believing a proposition $S_{0}$ is proportional to the maximal degree of coherence of sets $\mathcal{S}$ of propositions which $\mathcal{J}$ believes and which contain $S_{0}$.

Weak Graded Foundationalism can be seen as a version of Audi's [3] "mild foundationalism", and Weak Graded Coherentism is essentially what BonJour [6] defended; they are actually consistent with each other as Audi [3] himself remarked. Furthermore, in light of concern for holism - entailing an interest in very large belief systems exhibited by many coherentists, it is natural to try to wed Weak Graded Coherentism and Weak Graded Infinitism. Indeed, the objective in our formal analysis is to refute Strong Foundationalism and Strong Infinitism, while at the same time showing the consistency of Weak 
(Graded) Coherentism and Weak (Graded) Infinitism. One step towards this goal has already been reached in this section by showing that Weak Coherentism and Weak Infinitism are compatible.

\section{A formal probabilistic framework for analysing belief systems and their epistemic justification}

We shall now turn to a formal analysis of infinite belief systems (including regresses), inferential justification and (to some extent) coherence. We shall allow for partial beliefs and adopt the thesis of probabilism - thus formalising degrees of beliefs as probabilities. There is an ongoing, very lively and advanced discussion in formal epistemology about the optimal ways of defending probabilism, cf. e.g. Joyce [32, Easwaran and Fitelson [16], Fitelson and McCarthy [18], Leitgeb and Pettigrew [42, 43], Wedgwood [63]; reviewing this discussion would be way beyond the scope of this paper.

We choose, within these confines, a relatively simple and natural framework, which bears some resemblance to the approach in Carnap [11. For our purposes in this note, a belief system $\mathcal{S}$ will simply be a set of conditional probability assignments, interpreted as degrees of belief. In other words, we fix some algebra $\mathcal{A}$ of propositions, and we shall call a belief system a set of triples, denoted $\langle A \mid B \| \alpha\rangle$, where $A, B \in \mathcal{A}$ and the third and last entry $\alpha$ is a real number between 0 and 1, i.e. $\alpha \in[0,1]$. The expression $\langle A \mid B \| \alpha\rangle$ is to be read as "the belief system assigns to $A$, given $B$, a conditional degree of belief $\alpha$ ".

We adopt a semantic notion of probabilistic consistency. We call a belief system $\mathcal{S}$ probabilistically consistent if and only if there exists a probability measure $P: \mathcal{A} \rightarrow[0,1]$ such that $P(A \mid B)=\alpha$ whenever $\langle A \mid B \| \alpha\rangle \in \mathcal{S}$ for any $A, B \in \mathcal{A}$ and $\alpha \in[0,1]$. Such a probability measure $P$ is then said to support $\mathcal{S}$ or to be compatible with $\mathcal{S}$. The set of all probability measures on $\mathcal{A}$ that support a given belief system $\mathcal{S}$ will be denoted $\mathcal{P}_{\mathcal{S}}$.

A (recipe for a) probabilistic regress is a pair consisting of two sequences $\alpha, \beta \in[0,1]^{\mathbf{N}}$ ( $\mathbf{N}$ denoting the set of nonnegative integers), such that $\alpha_{k}>\beta_{k}$ for all $k \in \mathbf{N}$. The consistency notion for (recipes for) probabilistic regresses is then simply inherited from the probabilistic consistency notion for belief systems: A recipe for a probabilistic regress $\langle\alpha, \beta\rangle$ is called consistent if and only if there exists a sequence of propositions $S=\left\langle S_{k}\right\rangle_{k \in \mathbf{N}} \in \mathcal{A}^{\mathbf{N}}$ such that

$$
\left\{\left\langle S_{k} \mid S_{k+1} \| \alpha_{k}\right\rangle: k \in \mathbf{N}\right\} \cup\left\{\left\langle S_{k} \mid \complement S_{k+1} \| \beta_{k}\right\rangle: k \in \mathbf{N}\right\}
$$

is a probabilistically consistent belief system. (Herein, $\complement$ denotes the set-theoretic complement operator, so that $\complement A$ denotes the proposition/event 'non- $A$ '.) In other words, a probabilistic regress is consistent if and only if there exist both a sequence $S=\left\langle S_{k}\right\rangle_{k \in \mathbf{N}} \in \mathcal{A}^{\mathbf{N}}$ 
and a probability measure $P: \mathcal{A} \rightarrow[0,1]$ such that both

$$
0<P\left(S_{k+1}\right)<1
$$

and

$$
P\left(S_{k} \mid S_{k+1}\right)=\alpha_{k}>\beta_{k}=P\left(S_{k} \mid \boldsymbol{C} S_{k+1}\right)
$$

hold for all $k \in \mathbf{N}$; such a pair $\langle P, S\rangle$ will be called a model for the probabilistic regress $\langle\alpha, \beta\rangle$. This entails that, in the terminology of Bayesian epistemology (cf. e.g. Hájek and Hartmann [23]), $S_{k+1}$ confirms $S_{k}$ for all $k \in \mathbf{N}$ - so that $S_{0}$ is confirmed by $S_{1}$, which is confirmed by $S_{2}$, which is confirmed by $S_{3}$ etc. ad infinitum.

\section{In defense of (weak) infinitism: The consistency of infinite probabilistic regresses}

A recently established consistency theorem (Herzberg [27, 28]) asserts that a recipe for a probabilistic regress $\langle\alpha, \beta\rangle$ is consistent if and only if it is admissible in the sense that either

- $\prod_{k=0}^{\infty}\left(\alpha_{k}-\beta_{k}\right)>0$, or

- (1) $\beta_{k}>0$ for infinitely many $k$, and

(2) $\sum_{\ell=0}^{\infty} \beta_{\ell+k} \prod_{i=0}^{\ell-1}\left(\alpha_{i+k}-\beta_{i+k}\right)<1$ for all $k \in \mathbf{N}$.

This condition looks unnatural only at first sight. In fact, as Herzberg [27] shows, any violation of admissibility by a regress $\langle\alpha, \beta\rangle$ immediately would entail the following: If $S \in \mathcal{A}^{\mathbf{N}}$ and $P$ are such that $P\left(S_{k} \mid S_{k+1}\right)=\alpha_{k}$ and $\beta_{k}=P\left(S_{k} \mid \mathcal{\complement} S_{k+1}\right)$, then also $P\left(S_{k}\right)=0$ or $P\left(S_{k}\right)=1$ for some $k \in \mathbf{N} \backslash\{0\}$, whence either $P\left(S_{k} \mid S_{k+1}\right)$ or $P\left(S_{k} \mid \complement S_{k+1}\right)$ are no longer defined - and $\langle P, S\rangle$ cannot be a model of $\langle\alpha, \beta\rangle$.

Hence, this theorem establishes that there is a rich class of probabilistic regresses that are consistent. Moreover, if $\langle P, S\rangle$ is a model of $\langle\alpha, \beta\rangle$, then $P\left(S_{0}\right)=0$. This directly and formally refutes the two arguments against probabilistic infinitism that originate with Russell [59], who thought that $P\left(S_{0}\right)=0$ must hold for all probabilistic infinite regresses.5 and C.I. Lewis [44], who held probabilistic infinite regresses to be probabilistically inconsistent:

"[I]n the kind of case here in point, one is always on the wrong end of any segment of the series, always required to determine something else first before one can determine what one wants to determine. The supposition that the probability of anything

\footnotetext{
4In Peijnenburg's [50 or Peijnenburg and Atkinson's [51 terminology, confirmation is referred to as probabilistic support. Since this is not the standard Bayesian terminology and we use the verb 'to support' also in a different formal sense (viz. when referring to the set of probability measures supporting a belief system), we have chosen not to follow Peijnenburg's example on this minor point.

${ }^{5} \mathrm{He}$ was corrected by Reichenbach in a private letter and admitted his error, as explained by Peijnenburg and Atkinson [51, p. 338].
} 
whatever always depends on something else which is only probable itself, is flatly incompatible with the justifiable assignment of any probability at all." [44, p. 173] (emphasis inserted by this author)

(For a discussion of some historical aspects of infinitism, cf. Peijnenburg and Atkinson [51, 52.) In addition, the said consistency theorem can be used to counter more recent arguments to infinitism as well (cf. Herzberg [28]).

Thus, we may conclude that the well-known and historical arguments against infinite regresses of reasons fail; it is not so obvious that infinite regresses could not constitute epistemic justification. A quick dismissal of infinitism will not do. But what about the other extreme: Are there examples of regresses of reasons which clearly do not provide epistemic justification?

We shall now argue that not every probabilistic regress of reasons provides epistemic justification for the proposition that was the point of depart of the regress - even if it terminates (i.e. consists only of finitely many reasons)! To see this, we need to review the foundationalist Principle of Inferential Justification and how it is typically understood in a probabilistic setting (within the existing literature).

This probabilistic setting is, in principle, open to several interpretations, including both subjective and objective (such as evidential) Bayesian ones. In order to allow for variant interpretations of probability, we shall in the following not explicitly refer to the (conditional) probabilities as (conditional) degrees of belief. That being said, our intended interpretation is subjective Bayesian, in the sense that we like to think of the (conditional) probabilities below as (conditional) degrees of belief. In such a subjective Bayesian setting, believing an inequality involving conditional probabilities - as in the principles (BPIJ), (QBPIJ), (RPBIJ) below - is interpreted as having conditional degrees of belief that satisfy the respective inequalities.

\section{Common ground between strong foundationalism and strong infinitism: The Principle of Inferential Justification}

The Principle of Inferential Justification is usually rendered as follows (cf. Fumerton [20, 21] who employs a slightly different notation):

(PIJ) To be justified in believing $E$ on the basis of $F$ one must not only be (1) justified in believing $F$, but also (2) justified in believing that $F$ makes probable E.

By the Principle of Inferential Justification as a necessary and sufficient condition we mean the following assertion: 
(PIJ') One is justified in believing $E$ on the basis of $F$ if and only if one is (1) justified in believing $F$ and (2) justified in believing that $F$ makes probable $E$.

To be sure, in order to defend a version of foundationalism using a regress argument, one only needs (PIJ), i.e. the "only if" part of (PIJ') (cf. e.g. Fumerton [21 for a succinct presentation of the connection between the regress problem and PIJ). This caveat notwithstanding, if one aims at a fully-fledged theory of epistemic justification, one needs a sufficient condition as well - and for a staunchly inferentialist theory of epistemic justification (be it of foundationalist or infinitist stripe), the simplest candidate for such a principle would clearly be (PIJ'). Since we aim at setting up strong versions of foundationalism and infinitism for a dilemma, we will henceforth work with (PIJ').

Moreover, a prima facie natural interpretation of " $F$ makes probable $E$ " would be " $F$ confirms $E$ " in the sense of Bayesian confirmation theory (which perhaps more accurately should be called "Carnapian confirmation theory", as its modern roots are to be found in Carnap's Logical foundations of probability [10], cf. Maher [46]). Under this interpretation, (PIJ') becomes the following Bayesian Principle of Inferential Justification:

(BPIJ) One is justified in believing $E$ on the basis of $F$ if and only if one is (1) justified in believing $F$ and (2) justified in believing that $P(E \mid F)>P(E)$.

The last inequality is, of course, equivalent to the formula $P(E \mid F)>$ $P(E \mid \complement F){ }^{6}$ which in the terminology of Peijnenburg [50] or Peijnenburg and Atkinson [51, 52 is expressed as F probabilistically supports $E$. The treatment of regresses of inferential justification in Peijnenburg [50] as well as Peijnenburg and Atkinson [51, 52] suggests that at least the necessity part of a principle like (BPIJ) is not seen as extraordinarily controversial, neither by infinitists nor foundationalists that subscribe to some kind of probabilism. It is therefore not too far-fetched to suppose that strong foundationalists and infinitists that accept probabilism will be adherents of a principle like (BPIJ). Nevertheless, there are instances of (BPIJ) where the weight of support which the reason lends to the proposition in question is, while not zero, so meagre that it can hardly be said to afford "justification" for

\footnotetext{
${ }^{6}$ As a few elementary rearrangements show:$$
P(E \mid F)>P(E \mid \complement F) \Leftrightarrow \frac{P(E \cap F)}{P(F)}>\frac{P(E \backslash F)}{1-P(F)}
$$$$
\Leftrightarrow P(E \cap F)-P(E \cap F) P(F)>P(E \backslash F) P(F)
$$$$
\Leftrightarrow P(E \cap F)>P(E) P(F) \Leftrightarrow P(E \mid F)>P(E) \text {. }
$$ 
believing that proposition.7 Still, in their latest paper, Peijnenburg and Atkinson 53 clarify that they would only subscribe to the necessity part of a principle like (BPIJ) - i.e. a probabilistic reading of (PIJ) as opposed to (PIJ') - , not the sufficiency part. Thus, Peijnenburg and Atkinson [53] themselves are not susceptible to the criticism above. Nevertheless, any strong, anti-coherentist infinitist who (perhaps motivated by the surprising viability of infinite regresses of justification demonstrated by Peijnenburg, Atkinson and Herzberg) goes beyond Peijnenburg and Atkinson and accepts the sufficiency part of (BPIJ) as well will have to respond to the above challenge.

In the opinion of this author, whether a proposition is epistemically justified is actually a matter of degree rather than a binary decision. But this cannot be granted by adherents of a literal reading of the Principle of Inferential Justification.

A refuge for someone who wants to uphold the Principle of Inferential Justification and give it an interpretation that accommodates Bayesian confirmation theory might be the following more quantitative version of the Bayesian Principle of Inferential Justification:

(QBPIJ) One is justified in believing $E$ on the basis of $F$ if and only if one is (1) justified in believing $F$ and (2) justified in believing that $P(E \mid F)-P(E) \geq c$.

Herein, $c$ denotes a threshold for partial justification to become full justification - just like the Lockean thesis postulates a threshold for partial belief to turn into full belief $]^{8}$

One problem with (QBPIJ) lies in the indiscriminate use of the same $c$ for all candidate beliefs $E$, which means that it might turn out to be very hard or even impossible under (QBPIJ) to justify one's belief in an event with a relatively high prior probability given rigorous demands on what counts as confirmatory evidence. Suppose, for instance, a proposition $E$ has prior probability $P(E)=.81$ and we employ (QBPIJ) with $c=.2$. Then, in order to justify our belief in $E$, we would have to find a piece of confirmatory evidence such that $P(E \mid F)=.81+.2>1$, which is impossible!

\footnotetext{
7 On this point, I am indebted to an anonymous referee, who suggested the following example. Suppose there is a 1000 ticket lottery, $F$ is the event that ticket $\# 1000$ does not win, and $E$ is the event that ticket $\# 1$ wins. Suppose you learn from a reliable source that $F$ is true, so you are justified in believing it, and condition (1) in (BPIJ) is met. So is $(2): P(E \mid F)=1 / 999>1 / 1000$. But surely you are not justified in believing $E$ on the basis of $F$. In terms of Bayesian confirmation theory, the degree of confirmation for $E$ offered by $F$ (according to the relevance measure of confirmation) is minute, viz. just $1 / 999-1 / 1000$, which is of order $10^{-6}$.

8 A popular version of what is known as the Lockean thesis demands that in order for a proposition $S_{0}$ to be considered a full belief of an agent, that agent's degree of belief in $S_{0}$ must exceed a certain threshold. Locke's Essay contains several passages that suffer such an interpretation [45, Book IV, Chapters XIV-XVII].
} 
Another natural and apparently more easily defensible interpretation of the " $F$ makes $E$ probable" in Fumerton's (PIJ) and the stronger (PIJ') is that $P(E \mid F)$, the subjective probability of $E$ given $F$, be sufficiently high:

(RBPIJ) One is justified in believing $E$ on the basis of $F$ if and only if one is (1) justified in believing $F$ and (2) justified in believing that $P(E \mid F) \geq c$.

Regarding the use of the parameter $c,(\mathrm{RBPIJ})$ is more closely related to the Lockean thesis than (QBPIJ), because in both (RBPIJ) and the Lockean thesis, $c$ is simply a lower bound for the (conditional) subjective probability of a candidate belief.

In Section 7, we shall point to some serious concerns about (BPIJ), (QBPIJ) and (RBPIJ), and in Section 8, we will conclude that the strong Principle of Inferential Justification should be abandoned in a probabilistic setting. One might already wonder at this point whether we have overlooked an important alternative probabilistic Principle of Inferential Justification. For, in their aforementioned latest paper, Peijnenburg and Atkinson [53] ultimately hint at the following acceptance criterion in which only the necessity part of the Principle of Inferential Justification enters (so that Peijnenburg and Atkinson clearly distance themselves from the stronger (PIJ') and its problematic probabilistic formalisations (BPIJ), (QBPIJ) and (RBPIJ)):

(PBPIJ) One is justified in believing $E_{0}$ on the basis of $E_{1}$ and $E_{1}$ on the basis of $E_{2}$ etc. ad infinitum if and only if one is justified in believing $P\left(E_{k} \mid E_{k+1}\right)>$ $P\left(E_{k}\right)$ for all $k \in \mathbf{N}$ and, in addition, $P\left(E_{0}\right) \geq c$.

This principle is immune to the criticism that we shall offer in Section 7. However, the fundamental difference to (BPIJ), (QBPIJ) and (RBPIJ) is that (PBIPJ) conjoins a probabilistic version of (PIJ) (as opposed to (PIJ')) with a global threshold constraint. The principle (PBPIJ) therefore comes very close to an admission that epistemic justification should rather be seen as a property of whole belief systems than of individual beliefs. This, however, is already the first and arguably decisive step towards coherentism - exactly the account of epistemic justification that this paper seeks to revive in a new formalisation.

In other words, (PBIPJ) is not a very strong, anti-coherentist version of infinitism at all. What separates (PBPIJ) from the version of coherentism outlined in Section 9 below is (A) (PBPIJ)'s binary understanding of epistemic justification, (B) (PBPIJ)'s presupposition of a unique subjective probability measure, and (C) (PBPIJ)'s apparent disregard for coherence consideration. Now, (B) does not indicate a big divide, because one can easily generalise (PBPIJ) through universal quantification over all $P$ that are compatible with the belief 
system. Moreover, if (PBPIJ) is viewed as merely a rough, binary approximation of a more fine-grained notion of epistemic justification, (A) and (C) will not stand in the way of reconciling (PBPIJ) with the formalisation of graded coherentism sketched in Section 9 below.

Thus, we shall henceforth treat (PBPIJ), as it were, as an ally rather than a foe. For the remainder of the paper, we shall concentrate on a proposal for a refutation of the principles (BPIJ), (QBPIJ), (RBPIJ) and the subsequent elaboration of the main ingredients of a formal graded coherence notion.

\section{Problematic consequences of Bayesian variants of the Principle of Inferential Justification}

As we shall see presently, both foundationalist and infinitist accounts of epistemic justification based on the Bayesian Principle of Inferential Justification (BPIJ) face a serious conceptual difficulty: Sufficiently long finite as well as infinitely long chains of probabilistic justification may result in arbitrarily small probabilities for the proposition that is to be justified. To see this, suppose we are given propositions $E_{0}, \ldots, E_{n}$ such that for all $i<n, E_{i+1}$ confirms $E_{i}$ and let us introduce the abbrevations $\alpha_{i}:=P\left(E_{i} \mid E_{i+1}\right)$ and $\beta_{i}:=$ $P\left(E_{i} \mid \complement E_{i+1}\right)$. Then, by iterating an appropriate instance of the law of total probability,

$$
P\left(E_{0}\right)=P\left(E_{n}\right) \prod_{i=0}^{n-1}\left(\alpha_{i}-\beta_{i}\right)+\sum_{\ell=0}^{n-1} \beta_{\ell} \prod_{i=0}^{\ell-1}\left(\alpha_{i}-\beta_{i}\right)
$$

(cf. Peijnenburg and Atkinson [51, Equations (6)]). For the case of constant $\alpha_{i}, \beta_{i}$, Equation (11) reads:

$$
\begin{aligned}
P\left(E_{0}\right) & =P\left(E_{n}\right)(\alpha-\beta)^{n}+\sum_{\ell=0}^{n-1} \beta(\alpha-\beta)^{\ell} \\
& =P\left(E_{n}\right)(\alpha-\beta)^{n}+\beta \frac{1-(\alpha-\beta)^{n}}{1-\alpha+\beta} \\
& =\frac{\beta}{1-\alpha+\beta}+\left(P\left(E_{n}\right)-\frac{\beta}{1-\alpha+\beta}\right)(\alpha-\beta)^{n}
\end{aligned}
$$

(cf. Peijnenburg [50, Equation (12)]), and in the case of an infinite regress (i.e. $n \rightarrow \infty$ ), one has, of course,

$$
P\left(E_{0}\right)=\frac{\beta}{1-\alpha+\beta}
$$

(Peijnenburg [50, p. 601]). Therefore, keeping $\alpha<1$ fixed, one has $P\left(E_{0}\right) \longrightarrow 0$ as $\beta \rightarrow 0$. For instance, in the example of the recent paper by Peijnenburg and Atkinson [52, p. 119], $P\left(E_{0}\right)$ is as small as .2. Thus, an infinitist who applies the sufficiency part of (BPIJ) must claim that an epistemic subject is justified in believing a proposition to 
which she herself assigns a very small probability. This is very counterintuitive (and exhibits some formal similarity to the Sorites paradox). Even though there has recently been an attempt to vindicate a position that allows for justified belief in propositions to which a small credence is assigned, this vindication only refers to certain, very special scenarios (which may be seen as probabilistic analogues of Gettier examples).

The foundationalist who accepts the sufficiency part of (BPIJ) is only in a slightly better position: Equation (3) teaches us that for every fixed $n$ and fixed $\alpha<1$, one has $P\left(E_{0}\right) \longrightarrow 0$ as $\beta \rightarrow 0$. This holds regardless of the value of $P\left(E_{n}\right)$. Even if $E_{n}$ is a properly basic belief and thus assigned a probability of exactly 1 and $E_{0}$ has been obtained by five successive inferences (from the properly basic belief $\left.E_{n}\right)$, each of which was relatively robust, say, $\alpha=.6$ and $\beta=.2$, then $P\left(E_{0}\right)=\frac{1}{3}+\frac{2}{3} \times \frac{32}{3125}=\frac{1}{3} \times\left(1+\frac{64}{3125}\right)<\frac{1}{2}$. So, $E_{0}$ will be less likely than its negation; therefore, it is almost absurd to claim that $E_{0}$ instead of non- $E_{0}$ is justified!

As was mentioned above, abandoning (BPIJ) for (RBPIJ) goes against the consensus of the existing - rather small, as we readily admit - literature on infinitism in a probabilistic setting. But be this as it may, such a strategy would only be of little use to strong foundationalists or strong infinitists, because anyone who subscribes to (RBPIJ) rather than (BPIJ) faces a similar challenge to the one outlined in the preceding paragraph: If $E_{0}$ is made probable by $E_{1}$, which is made probable by $E_{2}$, etc., and we interpret " $F$ makes $E$ probable" according to (RBPIJ) as $P(E \mid F) \geq c$, then (RBPIJ) teaches that a candidate belief $E_{0}$ is justified if and only if there is a chain of reasons $E_{1}, E_{2}, \ldots$ such that $P\left(E_{k} \mid E_{k+1}\right) \geq c$ for all $k$; for a foundationalist, a chain of reasons ends by definition in some immediately justified belief $E_{n}$, and for an infinitist, a chain of reasons has to continue ad infinitum. In our previous notation, all that (RBPIJ) demands is that $\alpha_{k}=P\left(E_{k} \mid E_{k+1}\right) \geq c$ for all $k$; nothing is said about the sequence of conditional probabilities $\beta_{k}=P\left(E_{k} \mid \complement E_{k+1}\right)$. By choosing a sequence of events with constant $\beta_{k}=\beta<1-c 9^{9}$ we can ensure, in light of the general Equation (4), that

$$
P\left(E_{0}\right)=\frac{\beta}{1-c+\beta}<1 / 2,
$$

so that any infinitist subscribing to (RBPIJ) must claim that belief in propositions to which a subjective probability of less than $1 / 2$ is assigned is justified. And by choosing $\beta<1-c$ as well as a sufficiently large $n$, we can even ensure, in light of Equation (3), that the same is true for any foundationalist subscribing to (RBPIJ). Again, infinitists and foundationalists who take (RBPIJ) at face value must claim that

\footnotetext{
${ }^{9}$ The choice of such a sequence is possible in light of the consistency theorems for infinite regresses, cf. Section 5 .
} 
an agent is justified in believing some proposition $E_{0}$ to which she assigns subjective probability $<1 / 2$; but clearly, such an agent is, if anything, justified in believing the negation of that proposition!

\section{A dilemma for Bayesian foundationalists and infinitists}

In order to circumvent supporting the epistemic justification of beliefs whose content is less likely than its negation, an infinitist or foundationalist has, in principle, two options: She may either (a) reject the Bayesian interpretations of " $F$ makes probable $E$ " in (BPIJ) and (RBPIJ) or (b) take exception to the sufficiency part of either (BPIJ) or (RBPIJ).

The former way looks very much like an ad hoc solution. For, if neither Bayesian confirmation theory nor an adaptation of the Lockean thesis give a satisfactory explanation of " $F$ makes probable $E$ ", what does? Also, the Bayesian Principle of Inferential Justification (BIPJ) can be motivated by probability logic: It is a $p$-valid conclusion - in the sense of Adams' probability logic - to conclude from a very high probability for $B$ given $A$ ( $A \Rightarrow B$ in Adams' notation) and a very high probability for $A$ that $B$ must have a high probability (cf. Adams [1, p. 157f.]) as well. For short, dropping the two proposed Bayesian interpretations of " $F$ makes probable $E$ " does not seem to be, after all, a satisfactory option.

What remains is the latter possibility - to jettison the "if" part of (BPIJ) or (RBPIJ). This, however, is a rather thorny way out of the problem, too: If the sufficiency part of (BPIJ) or (RBPIJ) is incomplete, which additional conditions on the right-hand side of the Principle of Inferential Justification would be needed to obtain a sufficient criterion for inferential transfer of epistemic justification? Notice that in order to exclude the above examples of pseudojustification, these conditions must be of such a form that they entail quantitative lower bounds on $\beta$ or (in the case of the foundationalist) quantitative upper bounds on $n$. At least to this author, it is totally enigmatic how general epistemological principles could provide quantitative bounds of such a form. Furthermore, the appeal of the Principle of Inferential Justification - at least for non-coherentists, who conceive of epistemic justification as a property of individual beliefs one at a time, not of entire belief systems at once - lies precisely in the fact that it allows to transfer epistemic justification from one proposition to the other without examining how the former proposition was justified.

In sum, the Bayesian Principle of Inferential Justification, in both its revised (RBPIJ) and unrevised (BIPJ) forms, is on the one hand far too permissive as a necessary and sufficient criterion for epistemic justification. On the other hand, if our analysis is correct, there is no 
fix for this problem short of abandoning the Principle of Inferential Justification.

Having criticised the Principle of Inferential Justification and (in a previous section) foundationalist views of infinite regresses, the remainder of this paper is more positive. We shall propose some basic aspects of an alternative theory of epistemic justification along coherentist lines which nevertheless can accommodate some features of infinitism and even foundationalism.

\section{Toward a solution: Reviving coherentism}

So far, we have argued that the Principle of Inferential Justification has, when applied in a probabilistic setting, highly undesirable consequences. But is there an alternative?

For a coherentist, it has been clear from the beginning that the structure of epistemic justification cannot be accurately captured by the Principle of Inferential Justification. Epistemic justification is a property not of individual beliefs, but of entire belief systems. And if those belief systems are probabilistic, i.e. consist of conditional degrees of beliefs, as outlined in the formal framework above, then a belief will in general only be justified to a certain degree. Moreover, it is quite evident that a holistic and gradual understanding of epistemic justification is, at the very least, not totally foreign to epistemological infinitism (cf. e.g. Klein [35, p. 138]).

The reader of this article may object that formal epistemology of the last two decades, starting with the papers by Klein and Warfield [38, 39], has buried any hopes for coherentism 10 However, if belief systems are understood as thoroughly probabilistic (as in our framework), it turns out that many of the arguments purporting to show the impossibility of coherence measures, are no longer tenable; for, this literature always criticises attempts of measuring the coherence of a set of propositions (or of a binary assignment of truth values to propositional variables), rather than the coherence of a set of conditional degrees of belief (which, according to probabilism, correspond to conditional probability assignments and thus induce sets of probability measures). Moreover, even Bovens and Hartmann [8], while noted for their impossibility theorem for real-valued coherence measures, proposed a vector-valued coherence measure. It would clearly exceed the confines of a short paper to elaborate a fully-fledged coherence theory of epistemic justification, i.e. a theory which construes

\footnotetext{
${ }^{10}$ This may be all the more surprising since coherentism was, after all, the position of eminent analytic epistemologists such as Goodman [22], Hempel [25, 26, Neurath [48, 49, Quine [54, Reichenbach [55] and Sellars 60] to name but a few; among influential contemporary epistemologists, coherentism has had adherents such as van Fraassen [19, and Jeffrey 31 at the very least shared their opposition to foundationalism.
} 
a notion of coherence that is equivalent to epistemic justification. Nevertheless, we may sketch some of the formal aspects of such a theory (which is the subject of a monograph in preparation).

The following points have to be chiefly borne in mind: (i) Epistemic justification is a matter of degree (cf. even according to philosophers who reject coherentism such as, e.g., Roderick M. Chisholm 12 or Peter Klein [35, p. 138]): a belief system may be more or less epistemically justified. (ii) So is coherence: a belief system may be more coherent or less coherent (cf. e.g. Brendel [9]). While these points are widely appreciated, we also add the following: (iii) Because of its holistic understanding of epistemic justification, coherentism should primarily clarify what the degree of coherence of comprehensive belief systems (e.g. entire worldviews) is; by only looking at comprehensive belief systems, we may safely assume that the belief system under consideration contains spontaneous cognitive beliefs (in BonJour's [6, Chapter 6] sense) and will thus not be isolated from the external world, but responsive to input from outside. ${ }^{11}$

The aforementioned thesis of Weak Graded Coherentism - which may very well be consistent with mild forms of foundationalisms such as Robert Audi's "moderate foundationalism" [3, p. 233] - could also be formulated thus: If two belief systems $\mathcal{S}_{1}, \mathcal{S}_{2}$ make statements about the same conditional events, then $\mathcal{S}_{1}$ is epistemically more justified than $\mathcal{S}_{2}$ if $\mathcal{S}_{1}$ has a greater degree of coherence than $\mathcal{S}_{2}$. We shall now explain cursorily what it means formally for a comprehensive belief system to exhibit a greater degree of coherence than another comprehensive belief system.

According to BonJour [6, Section 5.3], (1) any degree of coherence can only be assigned to logically consistent belief systems, and the degree of coherence depends isotonically on (2) its degree of probabilistic consistency and (3) the number and strength of inferential connections in it, and antitonically on (4) its fragmentation into subsystems that are not mutually inferentially connected. He also adds as a further requirement that (5) unexplained anomalies should decrease the degree of coherence as "advisable"; this requirement seems, however, beyond any hopes of being formalised, whence we shall ignore this advice.

The other, and arguably much more essential, four requirements can be formalised as follows: (1) The logical consistency of a belief system is tantamount to the non-emptiness of the intersection of the propositions/events in the belief system. (2) The probabilistic consistency of a belief system can be measured in terms of the size of the set of probability measures supporting a belief system. Since this

\footnotetext{
${ }^{11}$ Reichenbach already proposed a solution to the isolation objection along these lines through the inclusion of "observation sentences" ([56, p. 159] and [55, p. 83]) or "protocol sentences" [4, p. 171].
} 
set will have a distinctive geometrical structure (viz. the intersection of several hyperplanes with a simplex), there is a canonical way of measuring its size through a vector-valued quantity, viz. the pair consisting of the Hausdorff dimension and the Hausdorff measure [24]. (3) The number of inferential connections can be measured in terms of graph-theoretic notions of connectivity; their strength can be measured using a confirmation function. (4) The fragmentation can be measured in terms of the number of components, i.e. maximal connected (proper) subgraphs.

Using Robinsonian [58] nonstandard analysis (more precisely, the Transfer Principle of nonstandard analysis), one can extend this realvector-valued coherence notion defined for finite belief systems to a hyperreal-vector-valued coherence notion defined for hyperfinite belief systems. Thus, one arrives at a coherence notion which is applicable to certain infinite belief systems defined, too. This class of infinite belief systems includes all belief systems on hyperfinite probability spaces. Bearing in mind the universality of such spaces (as rigorously formulated and established Fajardo and Keisler [17, building on earlier work, especially by Hoover and Keisler [29]), this is already a very substantial degree of generality.

This is, we think, a quite natural approach to a formalised coherence theory of epistemic justification, even though it requires much further explication. A more elaborate treatment will be presented in a forthcoming paper. The bottomline is that such a theory can be greatly simplified by confining the discussion (a) to comparative coherence and comparative epistemic justification and (b) to the comparison of comprehensive belief systems only.

Before the emergence of the literature on infinitism, one would have considered part (b) to come at an enormous cost, viz. that infinite belief systems must be considered as well — which may have a regress-like structure. But now it is enough to point to refer to the discussion on infinitism in order to dispel this objection. For instance, the consistency theorem for infinite regresses of probabilistic justification (see above and, in greater detail, Herzberg [27, 28]) provides us with a large set of concrete examples of infinite belief systems which are demonstrably probabilistically consistent. Moreover, an inspection of the original proof of Herzberg's [27] consistency theorem shows that for any admissible probabilistic regress, there is a model $\langle P, S\rangle$ that is also logically consistent in the sense that $\bigcap_{n} S_{n} \neq \varnothing .12$ Thus we

\footnotetext{
${ }^{12}$ Herein, $\varnothing$ denotes the minimal element of the algebra $\mathcal{A}$ of propositions, viz. the empty set when identifying each proposition in $\mathcal{A}$ with its extension, i.e. the set of worlds in which it holds.
} 
have found a large set of infinite belief systems which demonstrably do possess more than a minimal degree of coherence ${ }^{13}$

Before concluding, let us briefly summarise what has been achieved in this paper so far. We have shown in this paper, rigorously and in a simple and natural Bayesian framework: (i) infinite regresses generically constitute probabilistically consistent belief systems; (ii) even though probabilistically consistent, both finite and infinite regresses can fail to establish epistemic justification based on the Principle of Inferential Justification; (iii) there is hope for a formal coherence notion which assigns an important rôle to both (a) probabilistic consistency and (b) to the presence and the strength of inferential connections and which (c) can be adapted to be applicable to infinite belief systems, too. We may therefore conclude that the theses introduced as Weak Graded Coherentism and Weak Graded Infinitism above are even jointly consistent, while both Strong Infinitism and Strong Foundationalism are, at least in a Bayesian setting, very problematic on account of their use of the Principle of Inferential Justification. Moreover, our account of epistemic justification based on a formalisation of BonJour's [6] analysis of the concept of coherence can also accommodate certain foundationalist intuitions: Belief systems may, of course, include statements like $\langle A \mid \Omega \| \alpha\rangle$ for $\alpha$ arbitrarily close to (or even equal to) 1, ${ }^{14}$ and there will normally be no lack of possibilities to extend such a statement to a belief system with a high degree of coherence (which for a coherentist entails a high degree of epistemic justification).

Finally, our sketch of a formal coherentist account of epistemic justification only involves degrees of coherence and thus of justification - without any maximum or canonical threshold which could be used to introduce a context-independent definition of knowledge. Therefore, it is compatible even with very cautious approaches to epistemic justification - which treat most instances of what is commonly held to be epistemic justification as merely "provisional" (cf. Klein [36, p. 26]) or a mere "approximat[ion]" of epistemic justification (cf. lately even BonJour [7, pp. 62, 76]).

\section{Conclusion}

This paper is an attempt to show that mild versions of coherentism and infinitism can be - and should be - reconciled in order to arrive at a convincing philosophical position regarding epistemic justification.

\footnotetext{
${ }^{13}$ It is interesting in this connection that de Finetti considered probabilistic consistency to be such an important component of a coherence notion that he simply [13, 14, 15. defined coherence as probabilistic consistency.

${ }^{14}$ Herein, $\Omega$ denotes the maximal element of the algebra $\mathcal{A}$ of propositions, viz. the set of all possible worlds when identifying each proposition in $\mathcal{A}$ with its extension (the set of worlds in which it holds).
} 
Our critique of the natural Bayesian variant of the Principle of Inferential Justification demonstrates that the mere existence of an infinite chain of probabilistic inferences is not enough to provide epistemic justification. We argue that in order to avoid the undesirable consequences of the Principle of Inferential Justification in a probabilistic setting, infinitists should jettison that Principle and instead look for a holistic, coherentist understanding of epistemic justification. And indeed, there does exist some hope for formalising a defensible notion of coherence that could serve as a starting point for a revived holistic coherence theory of epistemic justification.

Moreover such a revived and revised version of coherentism can - and for at least three reasons should - accommodate infinitist intuitions as well. First, we have outlined an alternative way of introducing foundationalism and infinitism (which is no longer based on the epistemological regress argument) and shown that mild versions of those positions are not genuine rivals after all. Secondly, infinite regresses of probabilistic justification will often turn out to possess more than a minimal degree of coherence, in light of their generic probabilistic consistency. Thirdly, if coherentists take their holistic commitment seriously, they need to accept very large, even infinite belief systems.

\section{References}

[1] E.W. Adams. A primer of probability logic. With a foreword by Brian Skyrms, volume 68 of CSLI Lecture Notes. CSLI Publications, Stanford, CA, 1998.

[2] Aristotle, A.J. Jenkinson, and G.R.G. Mure. Prior analytics and Posterior analytics. Authorhouse, Bloomington, IN, 2006.

[3] R. Audi. Epistemology: A contemporary introduction to the theory of knowledge. Routledge, Oxford, 3 edition, 2011.

[4] J. Barnes. Aristotle's theory of demonstration. Phronesis, 14(2):123-152, 1969.

[5] M. Bergmann. What's NOT wrong with foundationalism. Philosophy and Phenomenological Research, 68:161-165, 2004.

[6] L. BonJour. The structure of empirical knowledge. Harvard University Press, Cambridge, MA, 1985.

[7] L. BonJour. The myth of knowledge. Philosophical Perspectives, 24(1):57-83, 2010.

[8] L. Bovens and S. Hartmann. Bayesian epistemology. Oxford University Press, Oxford, 2003.

[9] E. Brendel. Coherence theory of knowledge: A gradational account. Erkenntnis, 50(2-3):293-307, 1999.

[10] R. Carnap. Logical foundations of probability. University of Chicago Press, Chicago, IL, 1950.

[11] R. Carnap. The aim of inductive logic. In E. Nagel, P. Suppes, and A. Tarski, editors, Logic, methodology and philosophy of science, pages 303-318. Stanford University Press, Stanford, CA, 1962.

[12] R.M. Chisholm. Theory of knowledge. Prentice-Hall, Englewood Cliffs, NJ, 3rd edition, 1989. 
[13] B. de Finetti. La prévision: ses lois logiques, ses sources subjectives. Annales de l'Institut Henri Poincaré, 7(1):1-68, 1937.

[14] B. de Finetti. Teoria delle probabilità: sintesi introduttiva con appendice critica. Volumi primo e secondo. Nuova Biblioteca Scientifica Einaudi. Giulio Einaudi Editore, Turin, 1970.

[15] B. de Finetti. Theory of probability: a critical introductory treatment. Vol. 1. Translated by Antonio Machi and Adrian Smith, with a foreword by D. $V$. Lindley. Wiley Series in Probability and Mathematical Statistics. John Wiley \& Sons, London, New York, Sydney, 1974.

[16] K. Easwaran and B. Fitelson. An "evidentialist" worry about Joyce's argument for probabilism. Dialectica, 66(3):425-433, 2012.

[17] S. Fajardo and H.J. Keisler. Model theory of stochastic processes, volume 14 of Lecture Notes in Logic. A.K. Peters, Natick, MA, 2002.

[18] B. Fitelson and D. McCarthy. Steps toward a new foundation for subjective probability. Work in progress, Munich Center for Mathematical Philosophy, 2012.

[19] B.C. van Fraassen. Belief and the will. Journal of Philosophy, 81(5):235-256, 1984.

[20] R. Fumerton. Replies to my three critics. Philosophy and Phenomenological Research, 58:927-937, 1998.

[21] R. Fumerton. Foundationalist theories of epistemic justification. In E.N. Zalta, editor, The Stanford Encyclopedia of Philosophy (Summer 2010 Edition). 2010.

[22] N. Goodman. The structure of appearance. Harvard University Press, Cambridge, MA, 1951.

[23] A. Hájek and S. Hartmann. Bayesian epistemology. In J. Dancy, E. Sosa, and M. Steup, editors, A companion to epistemology, pages 93-106. Blackwell, Malden, MA, 2010.

[24] F. Hausdorff. Dimension und äußeres Maß. Mathematische Annalen, 79(12):157-179, 1918.

[25] C.G. Hempel. On the logical positivists' theory of truth. Analysis, 2(4):49-59, 1935.

[26] C.G. Hempel. Some remarks on 'facts' and propositions. Analysis, 2(6):93-96, 1935.

[27] F.S. Herzberg. The consistency of probabilistic regresses. A reply to Jeanne Peijnenburg and David Atkinson. Studia Logica, 94(3):331-345, 2010.

[28] F.S. Herzberg. The consistency of probabilistic regresses: Some implications for epistemological infinitism. Erkenntnis, 78(2):371-382, 2013.

[29] D.N. Hoover and H.J. Keisler. Adapted probability distributions. Transactions of the American Mathematical Society, 286:159-201, 1984.

[30] T.H. Irwin. Aristotle's First Principles. Oxford University Press, Oxford, 1988.

[31] R.C. Jeffrey. Probability and the art of judgment. Cambridge Studies in Probability, Induction, and Decision Theory. Cambridge University Press, Cambridge, 1992.

[32] J.M. Joyce. Accuracy and coherence: Prospects for an alethic epistemology of partial belief. In F. Huber and C. Schmidt-Petri, editors, Degrees of Belief, volume 342 of Synthese Library, pages 263-297. Springer, Dordrecht, 2009.

[33] P. Klein. Foundationalism and the infinite regress of reasons. Philosophy and Phenomenological Research, 58:919-925, 1998.

[34] P. Klein. Human knowledge and the infinite regress of reasons. Philosophical Perspectives, pages 297-325, 1999. 
[35] P. Klein. Infinitism is the solution to the regress problem. In M. Steup and S. Ernest, editors, Contemporary debates in epistemology, pages 131-140. Blackwell, Malden, M.A., 2005.

[36] P. Klein. How to be an infinitist about doxastic justification. Philosophical Studies, 134:25-29, 2007.

[37] P. Klein. Skepticism. In E.N. Zalta, editor, The Stanford Encyclopedia of Philosophy (Summer 2011 Edition). 2011.

[38] P. Klein and T.A. Warfield. What price coherence? Analysis, 54(3):129-132, 1994.

[39] P. Klein and T.A. Warfield. No help for the coherentist. Analysis, 56(2):118$121,1996$.

[40] J. Kvanvig. Coherentist theories of epistemic justification. In E.N. Zalta, editor, The Stanford Encyclopedia of Philosophy (Summer 2011 Edition). 2011.

[41] K. Lehrer. Theory of knowledge. Westview Press, Boulder, CO, 2000.

[42] H. Leitgeb and R. Pettigrew. An objective justification of Bayesianism I. Measuring inaccuracy. Philosophy of Science, 77(2):201-235, 2010.

[43] H. Leitgeb and R. Pettigrew. An objective justification of Bayesianism II. The consequences of minimizing inaccuracy. Philosophy of Science, 77(2):236-272, 2010.

[44] C.I. Lewis. The given element in empirical knowledge. Philosophical Review, 61:168-172, 1952.

[45] J. Locke. An essay concerning human understanding. Ed. by. P.H. Nidditch. Oxford University Press, Oxford, 1979.

[46] P. Maher. Subjective and objective confirmation. Philosophy of Science, 63(2):149-182, 1996.

[47] P. Murphy. Coherentism in epistemology. In Internet Encyclopedia of Philosophy. 2006.

[48] O. Neurath. Soziologie im Physikalismus. Erkenntnis, 2:393-431, 1931.

[49] O. Neurath. Protokollsätze. Erkenntnis, 3:204-214, 1932.

[50] J. Peijnenburg. Infinitism regained. Mind, 116(463):597-602, 2007.

[51] J. Peijnenburg and D. Atkinson. Probabilistic justification and the regress problem. Studia Logica, 89(3):333-341, 2008.

[52] J. Peijnenburg and D. Atkinson. Grounds and limits: Reichenbach and foundationalist epistemology. Synthese, 181(1):113-124, 2011.

[53] J. Peijnenburg and D. Atkinson. The emergence of justification. Philosophical Quarterly, 63(252):546-564, 2013.

[54] W.V.O. Quine and J. Ullian. The web of belief. Random House, New York, 1970.

[55] H. Reichenbach. Experience and prediction. University of Chicago Press, Chicago, IL, 1938.

[56] H. Reichenbach. Are phenomenal reports absolutely certain? Philosophical Review, 61(2):147-159, 1952.

[57] N. Rescher. Foundationalism, coherentism, and the idea of cognitive systematization. Journal of Philosophy, 71(19):695-708, 1974.

[58] A. Robinson. Non-standard analysis. North-Holland, Amsterdam, 1966.

[59] B. Russell. Human knowledge. George Allen and Unwin, London, 1948.

[60] W. Sellars. Science, perception, and reality. Humanities Press, New York, 1963.

[61] W. Spohn. How to understand the foundations of empirical belief in a coherentist way. Proceedings of the Aristotelian Society, 98(1):23-40, 1998.

[62] E. Stump. Aquinas on the foundations of knowledge. Canadian Journal of Philosophy, supplementary volume 17:125-158, 1991.

[63] R. Wedgwood. Outright belief. Dialectica, 66(3):309-329, 2012. 
Munich Center for Mathematical Philosophy, Ludwig Maximilian University of Munich, Faculty of Philosophy, Philosophy of Science and Study of Religion, Chair of Logic and Philosophy of Language, Geschwister-Scholl-Platz 1, D-80539 Munich, Germany

E-mail address: frederik.herzberg@lrz.uni-muenchen.de

Center for Mathematical Economics, Bielefeld University, Universitätsstrasse 25, D-33615 Bielefeld, Germany

E-mail address: fherzberg@uni-bielefeld.de 